\title{
SOME THEOREMS ON THE FOURIER ANALYSIS OF POSITIVE DEFINITE FUNCTIONS ${ }^{1}$
}

\author{
L. SCHMETTERER
}

1. The main purpose of this paper is to prove some theorems about conjugate Fourier series, and similar orthogonal expansions, of a positive definite function. At the end of the paper there is given a slight generalization of the well-known continuity theorem for characteristic functions.

It was proved several years ago by Dugue [1] that if $\phi$ is a measurable positive-definite function then the Fourier series of $\phi$ is uniformly convergent.

Dugué's Theorem was actually stated in terms of characteristic functions, but it is equivalent to the present formulation on account of the Bochner-Khintchine Representation Theorem. More precisely one can state:

Theorem A (Dugué). Let $\phi$ be a measurable positive-definite function and suppose that $I$ is any interval of length $2 \pi$. Let $\phi_{1}$ be the restriction of $\phi$ to $I$ and $\phi^{*}$ the periodic extension of $\phi_{1}$ on the real line. Then, the Fourier series of $\phi^{*}$ is uniformly convergent in every compact subinterval of $I$. Since $\phi^{*}$ is in general not continuous at the endpoints of $I$, nothing more can be proved in this direction.

A well-known consequence of Theorem $\mathrm{A}$ is that the conjugate Fourier series of $\phi^{*}$ converges almost everywhere, and that

$$
\lim _{t \rightarrow 0} \frac{1}{\pi} \int_{e}^{\pi} \frac{1}{2} \cot \frac{1}{2} t\left[\phi^{*}(x+t)-\phi^{*}(x-t)\right] d t
$$

exists for almost all $x$ in $I$ and that this limit is the limit of the conjugate Fourier series almost everywhere [2, p. 269].

2. The following simple supplement to Dugués result can be proved:

Theorem I. Let I be the interval $(0,2 \pi)$ and let $\phi^{*}$ be the above defined function. The limit (1) exists for every $x \in I$. Furthermore, the conjugate Fourier series of $\phi^{*}$ converges uniformly in every compact subinterval of $I$ to the limit (1).

Received by the editors March 9, 1964 and, in revised form, October 12, 1964.

1 This work was done with the support from National Science Foundation Grant GP-96. 
Proof. Let $J$ be any compact subinterval of $I$. Let us write

$$
g_{n}(x, t)=(2 \pi)^{-1}\left(\cot \frac{1}{2} t\right)(1-\cos n t)\left[\phi^{*}(x+t)-\phi^{*}(x-t)\right] \text {. }
$$

It must be shown that $\int_{0}^{\pi} g_{n}(x, t) d t$ converges uniformly to the limit (1) for $x \in J$ as $n \rightarrow \infty$. Define $2 \delta=\min \left(\max _{x \in J} x, \max _{x \in J}(2 \pi-x)\right)$. Note that $\delta>0$. Obviously $\int_{\delta}^{\pi} g_{n}(x, t) d t$ converges uniformly for all $x \in J$ to $(2 \pi)^{-1} \int_{\delta}^{\pi}\left[\phi^{*}(x+t)-\phi^{*}(x-t)\right] \cot \frac{1}{2} t d t$. For $0 \leqq|t| \leqq \delta$ and $x \in J, \phi^{*}(x+t)$ can be represented in the form $\phi^{*}(x+t)$ $=\int_{-\infty}^{+\infty} e^{i(x+t) z} d F(z)$ where $F$ is a distribution function. For all natural numbers $n$, and for the set of all real numbers $z$,

$$
\int_{0}^{\delta} \sin t z \cot \frac{1}{2} t \cos n t d t
$$

is uniformly bounded; for every real $z$ this integral converges to 0 as $n \rightarrow \infty$. It follows that $\int_{0}^{\delta} g_{n}(x, t) d t$ converges uniformly to

$$
\frac{1}{\pi} \int_{-\infty}^{+\infty} e^{i x z} \int_{0}^{\delta} \sin t z \cot \frac{1}{2} t d t d F(z) \text {. }
$$

Furthermore, $\int_{\epsilon}^{\delta} \sin t z \cot \frac{1}{2} t d t$ is boundedly convergent, as $\epsilon \rightarrow 0$, in the interval $-\infty<z<\infty$. Therefore, it follows immediately that (2) is equal to

$$
\lim _{\epsilon \rightarrow 0}(2 \pi)^{-1} \int_{e}^{\delta} \cot \frac{1}{2} t\left[\phi^{*}(x+t)-\phi^{*}(x-t)\right] d t .
$$

This proves the theorem.

The question was raised by E. Lukacs and A. Rényi as to whether similar convergence statements can be made if the trigonometric system is replaced by other orthogonal systems. Since a characteristic function is continuous and bounded, a positive answer to this question can be given with the help of well-known equiconvergence theorems [3, p. 237].

It may for instance be proved that the expansion of a characteristic function in a series of Hermite-polynomials is convergent in every finite interval. Similarly it may be shown that the expansion of a characteristic function in a series of Jacobi-polynomials is convergent in the open interval $(-1,1)$. This contains as a special case a convergence statement about the expansion in terms of Legendrepolynomials. Of course, this last statement does not cover the more interesting, and nontrivial, question concerning the behaviour of the expansion of a characteristic function in a series of Legendre-polynomials about the singular points \pm 1 . In this direction we can prove 
THEOREM II. The expansion of a characteristic function in terms of Legendre-polynomials converges in the closed interval $[-1,1]$.

PRoof. Let $P_{n}, n \geqq 1$, be the $n$th Legendre polynomial defined over $[-1,1]$. Let $\phi$ be the characteristic function of a distribution function $F$. Define

$$
\begin{gathered}
a_{n}=\frac{1}{2}(2 n+1) \int_{-1}^{1} \phi P_{n} d x, \quad n=1,2, \ldots, \\
s_{n}(x)=\frac{1}{2}(n+1) \int_{-1}^{1} \frac{P_{n}(x) P_{n+1}(y)-P_{n+1}(x) P_{n}(y)}{y-x} \phi(y) d y, \\
n=1,2, \cdots, x \in[-1,1] .
\end{gathered}
$$

The latter integral is the $n$th partial sum of the development of $\phi$ in a Legendre series. We will show that $\lim _{n \rightarrow \infty} s_{n}(1)=\int_{-\infty}^{+\infty} e^{i z} d F(z)$. Exactly the same method of proof will lead to an analogous result for the point -1 .

It is easily seen $[4$, p. 33] that

$$
(n+1) \frac{P_{n}(x)-P_{n+1}(x)}{1-x}=P_{n}^{\prime}(x)+P_{n+1}^{\prime}(x) \quad \text { for every } x \in(-1,1) \text {. }
$$

It follows that

$$
s_{n}(1)=\frac{1}{2} \int_{-1}^{1}\left(P_{n}^{\prime}(y)+P_{n+1}^{\prime}(y)\right) \phi(y) d y ;
$$

hence from Fubini's Theorem

$$
s_{n}(1)=\frac{1}{2} \int_{-\infty}^{+\infty} \int_{-1}^{1}\left(P_{n}^{\prime}(y)+P_{n+1}^{\prime}(y)\right) e^{i y z} d y d F(z) .
$$

Now, it is easy to show [5, p. 117] that for every real $z$ and $n \geqq 1$ the following formula is correct:

$$
\int_{-1}^{1} P_{n}(x) e^{i z x} d x=i^{n}(2 \pi / z)^{1 / 2} J_{n+1 / 2}(z)
$$

where $J_{k}$ is the Bessel function with index $k$. For $z=0$ the right side of (4) is defined to be 0 . Using a partial integration with $P_{n}(1)=1$ and $P_{n}(-1)=(-1)^{n}$, we obtain

$$
\int_{-1}^{1} P_{n}^{\prime}(x) e^{i z x} d x=e^{i z}-(-1)^{n} e^{-i s}-i z \int_{-1}^{1} P_{n}(x) e^{i z x} d x
$$

for every real $z$ and $n \geqq 1$. It follows from (3), (4) and (5) that 


$$
\begin{aligned}
s_{n}(1)= & \frac{1}{2} \int_{-\infty}^{\infty}\left[\left(e^{i z}-(-1)^{n} e^{-i z}\right)+\left(e^{i z}-(-1)^{n+1} e^{-i z}\right)\right] d F(z) \\
& -\frac{1}{2} i^{n+1}(2 \pi)^{1 / 2} \int_{-\infty}^{\infty}\left[z^{1 / 2} J_{n+1 / 2}(z)+i z^{1 / 2} J_{n+3 / 2}(z)\right] d F(z) .
\end{aligned}
$$

The desired result follows if it can be shown that as $n \rightarrow \infty$,

$$
\int_{-\infty}^{\infty} z^{1 / 2} J_{n+1 / 2}(z) d F(z)=o(1) .
$$

It is well known that for large $z, z^{1 / 2} J_{n+1 / 2}(z)=O(1)$ uniformly in $n$. On the other hand, using for instance the formula

$$
J_{k}(z)=\frac{2(z / 2)^{k}}{\pi^{1 / 2} \Gamma(k+1 / 2)} \int_{0}^{\pi / 2} \cos (z \cos x) \sin ^{2 k} x d x,
$$

which is valid for $k>0$ and $-\infty<z<\infty$, it turns out that $\left|J_{k}(z)\right|$ $\leqq \pi^{1 / 2}(|z| / 2)^{k}(\Gamma(k+1 / 2))^{-1}$. Therefore, $\lim _{k \rightarrow \infty} J_{k}(z)=0$ for every real $z$, and the convergence is uniform in every compact interval. Statement (6) follows from this.

3. Another application of the Fourier analysis of characteristic functions leads to a slightly more general formulation of the continuity theorem. This generalization appears quite natural, and its proof is perhaps simpler than that of the more familiar formulation (see for example [6, p. 267]).

TheOREM III. Let $\phi_{1}, \phi_{2}, \cdots$ be a sequence of characteristic functions which converges in every finite interval to a limit $\phi$. If the Fourier series of $\phi$ at the point 0 is Cesdro-summable to 1 , then $\phi$ is a characteristic function, and conversely.

Proof. The last part of the theorem is a trivial consequence of Fejér's Theorem. For the first part, let $\left\{F_{i}\right\}$ be the sequence of distribution functions which corresponds to the sequence $\left\{\phi_{i}\right\}$. According to one of Helly's theorems, there exists a subsequence $\left\{F_{n_{i}}\right\}$ of $\left\{F_{i}\right\}$ which converges to a nondecreasing function $F$. Suppose $F$ has variation less than 1 . One can use the well-known formula

$$
\int_{0}^{x}\left(F_{n_{i}}(y)-F_{n_{i}}(-y)\right) d y=\frac{1}{\pi} \int_{-\infty}^{\infty} \frac{1-\cos x t}{t^{2}} \phi_{n_{i}}(t) d t,
$$

valid for every $x>0$. Since $F_{n_{i}}$ is nondecreasing, it follows easily that

$$
F_{n_{i}}(x)-F_{n_{i}}(-x) \geqq \frac{2}{\pi} \int_{-\infty}^{\infty} \frac{\sin ^{2}(t / 2)}{t^{2}} \phi_{n_{i}}(t / x) d t \quad \text { for every } x>0 \text {. }
$$


As $n_{i} \rightarrow \infty$, we find from the dominated convergence theorem that

$$
F(x)-F(-x) \geqq \frac{2}{\pi} \int_{-\infty}^{\infty} \frac{\sin ^{2}(t / 2)}{t^{2}} \phi(t / x) d t
$$

for all continuity points $x>0$ of $F$.

It follows from the assumption and from (7) that there exists a positive real number $d<1$ such that

$$
d \geqq \frac{2}{\pi} \int_{-\infty}^{\infty} \frac{\sin ^{2}(t / 2)}{t^{2}} \phi(t / x) d t
$$

uniformly for $x>0$. However, from the assumption that the Fourier series of $\phi$ at 0 is Cesàro-summable to 1 there exists a $\delta>0$ such that

$$
\frac{2}{\pi} \int_{-\delta(n+1)}^{\delta(n+1)} \phi(t /(n+1)) \frac{\sin ^{2}(t / 2)}{t^{2}} d t \rightarrow 1 \quad \text { as } n \rightarrow \infty
$$

and this contradicts (8).

CoROLlary 1. The continuity theorem is valid if $\phi$ is continuous at 0.

COROLLARY 2. The continuity theorem is valid if $\phi$ satisfies the condition

$$
\lim _{t \rightarrow 0} \frac{1}{2} t^{-1} \int_{-t}^{t} \phi(x) d x=1 .
$$

This follows from the fact that (9), and the condition

$$
\int_{-t}^{t}|\phi(x)| d x=O(t)
$$

for small $t$, together imply the Cesàro-summability to 1 of the Fourier series of $\phi$ at 0 . Condition (10) is of course satisfied.

REMARK. Theorem III follows also from the proof of the complete convergence theorem in Loève [7, p. 191]. This method leads to an even more general result: namely, the statement of Theorem III remains true if Cesàro-summability is replaced by any summability method which has the property that the Fourier series of a continuous function $f$ is summable to $f(x)$ at $x$.

I am indebted to one of the referees for bringing this remark to my attention.

\section{REFERENCES}

1. D. Dugué, Sur l'approximation d'une fonction caracteristique par sa strie de Fourier, C. R. Acad. Sci. Paris 244 (1957), 715-717. 
2. A. Zygmund, Trigonometric series, 2nd ed., Vol. I, Cambridge Univ. Press, Cambridge, 1959.

3. G. Szegö, Orthogonal polynomials, Amer. Math. Soc. Colloq. Publ. Vol. 23, Amer. Math. Soc., Providence, R. I., 1939.

4. A. Hobson, The theory of spherical and ellipsoidal harmonics, Cambridge Univ. Press, Cambridge, 1931.

5. E. Madelung, Die mathematischen Hilfsmittel des Physikers, 6th ed., Springer, Berlin, 1957.

6. A. Rényi, Wahrscheinlichkeitsrechnung, VEB Deutscher Verlag der Wissenschaften, Berlin, 1962.

7. M. Loève, Probability theory, Van Nostrand, New York, 1955.

MATHEMATISCHES INSTITUT DER UNIVERSITÄT WIEN AND Statistical Laboratory, The Catholic University

\title{
A NOTE ON THE HAUSDORFF MOMENT PROBLEM
}

\author{
C. W. LEININGER
}

In [1, pp. 630-635], J. H. Wells presented a solution of the Hausdorff moment problem for the case of a quasicontinuous mass function. The purpose of this note is to extend that result to include Riemann-integrable mass functions.

If $\left\{d_{n}\right\}$ is a number sequence, let $A_{n p}=\left(\begin{array}{l}n \\ p\end{array}\right) \Delta^{n-p} d_{p}, n \geqq p, p=0,1$, $2, \cdots$ We observe that $[1$, p. 634 , Theorem $2.4($ ii) (b) $]$ may be stated as follows:

If $\epsilon>0$, there is a finite collection $C$ of nonoverlapping subsegments $(u, v)$ of the segment $(0,1)$ such that $\sum_{c}(v-u)=1$ and if $u<y<z$ $<v$, then there is a positive integer $N$ such that if $n>N$, $\left|\sum_{n y<p \leq n z} A_{n p}+\sum_{n y \leq p<n z} A_{n p}\right|<\epsilon$.

The arguments used to establish $[1$, p. 634, Theorem 2.4] and the associated theorems and lemmas [1, pp. 630-633] are readily modified to supply a proof of the following theorem.

THEOREM. If $\left\{d_{n}\right\}$ is a number sequence, the following two statements are equivalent:

(i) There is a function $g$ Riemann-integrable on $[0,1]$ such that $d_{n}=\int_{[0,1]} I^{n} d g, n=0,1,2, \cdots$;

Received by the editors October 15, 1964. 